\title{
Sección traumática completa del istmo aórtico: Tratamiento tardío con reparación abierta. Reporte de un caso
}

\author{
Traumatic rupture of the aortic isthmus: Delayed treatment and open repair. Case report \\ Elizabeth Aguilar-Alaníz ${ }^{*}$, Rodrigo Reyes-Pavón², José A. Ruiz-Romero y Javier Esparza-Pantoja1 \\ ${ }^{1}$ Departamento de Cirugía Cardiovascular, Centenario Hospital Miguel Hidalgo; ${ }^{2}$ Centro de Ciencias de la Salud, Universidad Autónoma de \\ Aguascalientes. Aguascalientes, México.
}

La ruptura postraumática de la aorta se considera una lesión potencialmente letal, ya que aproximadamente el 70 a $80 \%$ de las víctimas mueren en la escena, y de los sobrevivientes, el $30 \%$ fallecerá en las siguientes 24 horas. Las causas más frecuentes incluyen accidentes de tráfico de alta velocidad, explosiones, caídas de alturas considerables y contusiones graves ${ }^{1}$.

Se ha propuesto una clasificación de la las lesiones aórticas, donde el tipo I se refiere a un desgarro intimal, el tipo II a un hematoma intramural, el tipo III a un seudoaneurisma y el tipo IV a una ruptura con extravasación activa².

De acuerdo al Registro Alemán de Trauma ${ }^{1}$, se calcula una prevalencia del $1.28 \%$, mientras que la incidencia de hospitalización por lesiones aórticas oscila entre el 2.5 y el 4.5 por 1,000,000 personas-años, y son más frecuentes las lesiones de tipo I (aproximadamente el 51.6\%), según el Registro Nacional Canadiense de Trauma; ; además, las lesiones asociadas con mayor frecuencia son las fracturas ortopédicas (68\%), seguidas por lesiones torácicas $(50.5 \%)$, y luego lesiones abdominales (41.3\%), específicamente lesiones hepáticas y esplénicas ${ }^{4}$.
Se presenta el caso de un trauma aórtico contuso manejado de forma conservadora al inicio, con la posterior reparación definitiva abierta a las 24 horas.

\section{Caso clínico}

Paciente masculino de 29 años de edad, que es atropellado por un vehículo automotor a alta velocidad. A su ingreso a Urgencias se encuentra traumatismo craneoencefálico leve (12 puntos en la escala de coma de Glasgow), fractura tibioperonea diafisiaria distal derecha expuesta, y trauma cerrado de tórax. Sus signos vitales eran: presión arterial 130/90, frecuencia respiratoria de 22 y frecuencia cardíaca de 87 . Sus pruebas de laboratorio mostraron una hemoglobina de $17 \mathrm{~g} / \mathrm{dl}$, un hematocrito de $53.1 \%$, plaquetas de 288,000 células $/ \mathrm{mm}^{3}$ y creatinina de $1.02 \mathrm{mg} / \mathrm{dl}$.

La radiografía de tórax mostró ensanchamiento mediastinal, desviación traqueal derecha y ensanchamiento del botón aórtico.

La tomografía toracoabdominal (Fig. 1) reveló un neumotórax derecho, derrame pleural izquierdo y, como hallazgos aórticos, desgarros intimales proximal y distal a la emergencia de la arteria subclavia

\section{Correspondencia:}

*Elizabeth Aguilar-Alaníz

Departamento de Cirugía Cardiovascular

Centenario Hospital Miguel Hidalgo

Avenida Gómez Morín

Col. La estación Alameda

Fecha de recepción: 18-07-2018

Disponible en internet: 06-05-2019

C.P. 2259, Aguascalientes, México

Fecha de aprobación: 14-12-2018

DOI: 10.24875/ACM.M19000039

Arch Cardiol Mex. 2019;89(3):275-278

www.archivoscardiologia.com

1405-9940 @ 2018 Instituto Nacional de Cardiología Ignacio Chávez. Publicado por Permanyer México SA de CV. Este es un artículo Open Access bajo la licencia CC BY-NC-ND (http://creativecommons.org/licenses/by-nc-nd/4.0/). 
izquierda, que delimitan la imagen de seudoaneurisma, así como hematoma periaórtico. Además de una contusión renal derecha de grado II, contusión renal izquierda de grado III y un hematoma retroperitoneal derecho.

El tratamiento interdisciplinario incluyó la colocación de una sonda endopleural izquierda por el Servicio de Cirugía General, por la que inicialmente se drenó $120 \mathrm{ml}$ de sangre, así como inmovilización de la fractura por traumatología. Por los hallazgos en la tomografía, se inicia manejo médico hipotensor con betabloqueador (metoprolol $50 \mathrm{mg}$ cada $12 \mathrm{~h}$ ), con lo que se mantiene una presión arterial sistólica menor a 120 y una frecuencia cardíaca menor a 60 durante 24 horas, tras lo cual pasa a quirófano para reparación quirúrgica.

El tratamiento quirúrgico definitivo consistió en toracotomía posterolateral izquierda, con bypass izquierdo (de aurícula izquierda a arteria femoral izquierda), y se encontró una sección total tipo III a nivel del ligamento arterioso, contenida por la pleura parietal, por lo que se realiza pinzamiento aórtico proximal y distal, se secciona el segmento afectado y se interpone un injerto de Woven Dacron de $22 \mathrm{~mm}$ de ancho y $10 \mathrm{~cm}$ de longitud, con un sangrado de $1,000 \mathrm{ml}$ y una duración de pinzamiento aórtico de $132 \mathrm{~min}$, tras lo cual sufre un paro cardíaco que requirió masaje cardíaco directo durante $33 \mathrm{~min}$, luego de esto recupera los signos vitales y se retira el bypass izquierdo. Se decide su ingreso en la Unidad de Terapia Intensiva, donde tiene una evolución inmediata tórpida, ya que desarrolla síndrome de insuficiencia respiratoria aguda y rabdomiólisis (con elevación de CPK hasta 10,934). Presenta mejoría de ambos cuadros 4 días después, por lo que se logra su extubación y egreso a piso, donde se realiza el tratamiento definitivo de la fractura mediante reducción abierta y enclavado centromedular.

Se le realiza tomografía de control (Fig. 2) que muestra un injerto interpuesto en el istmo aórtico, y reabsorción del hematoma periaórtico, sin dilatación aórtica proximal o distal al injerto.

A los 4 días evoluciona con un derrame pleural izquierdo loculado y restricción de la mecánica respiratoria, y se decide su manejo mediante toracoscopia con colocación de una sonda endopleural drenando $300 \mathrm{ml}$ de líquido seroso. La sonda se retira 3 días después, y se decide su egreso a domicilio. En el seguimiento a 8 meses de la cirugía, el paciente se encuentra asintomático y con una recuperación completa.

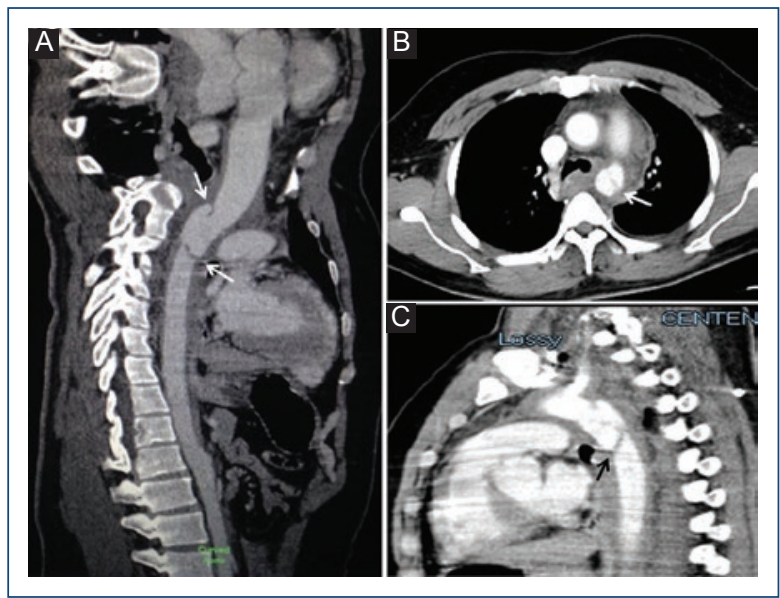

Figura 1. Angiotomografía helicoidal con contraste en fase arterial. A: Reconstrucción curva de la aorta (en su eje longitudinal), que muestra desgarros intimales (flechas) proximal y distal a la emergencia de la arteria subclavia izquierda, que delimitan la imagen de seudoaneurisma. B: Corte axial a nivel de la bifurcación traqueal. Se observa hematoma mediastinal y periaórtico (imagen hipodensa, flecha), y desgarro intimal en la aorta descendente torácica. C: Corte sagital. Desgarros intimales (flecha) e imagen de seudoaneurisma.

\section{Discusión}

El estudio de gabinete que se usa de manera rutinaria en nuestro centro de atención (el único en el estado con servicio de cirugía cardiotorácica) es la tomografía contrastada de tórax, que actualmente se considera el método de elección para cribado y diagnóstico de las lesiones por trauma contuso ${ }^{4,5}$.

El momento óptimo para intervenir la lesión aórtica y la decisión de atenderla antes o después de las lesiones asociadas ha sido un tema de debate por largo tiempo. Tomando en cuenta que el riesgo de ruptura de una lesión aórtica por trauma es más alto en las primeras 24 horas del accidente, la atención inmediata se consideró como el estándar de atención por décadas. Sin embargo, después de la publicación del artículo de Fabian, et al. ${ }^{6}$ en 1998, el uso de esquemas antihipertensivos se generalizó. El éxito de estos esquemas para prevenir la ruptura aórtica ha resultado en la práctica en retrasar las reparaciones de las lesiones en la aorta por trauma tanto en pacientes de alto como de bajo riesgo. En el 2014, la Eastern Association for the Surgery of Trauma ${ }^{7}$ desarrolló la actualización de sus guías para diagnóstico y manejo del daño aórtico por trauma cerrado de tórax. Siete estudios fueron incluidos en su análisis, y se 


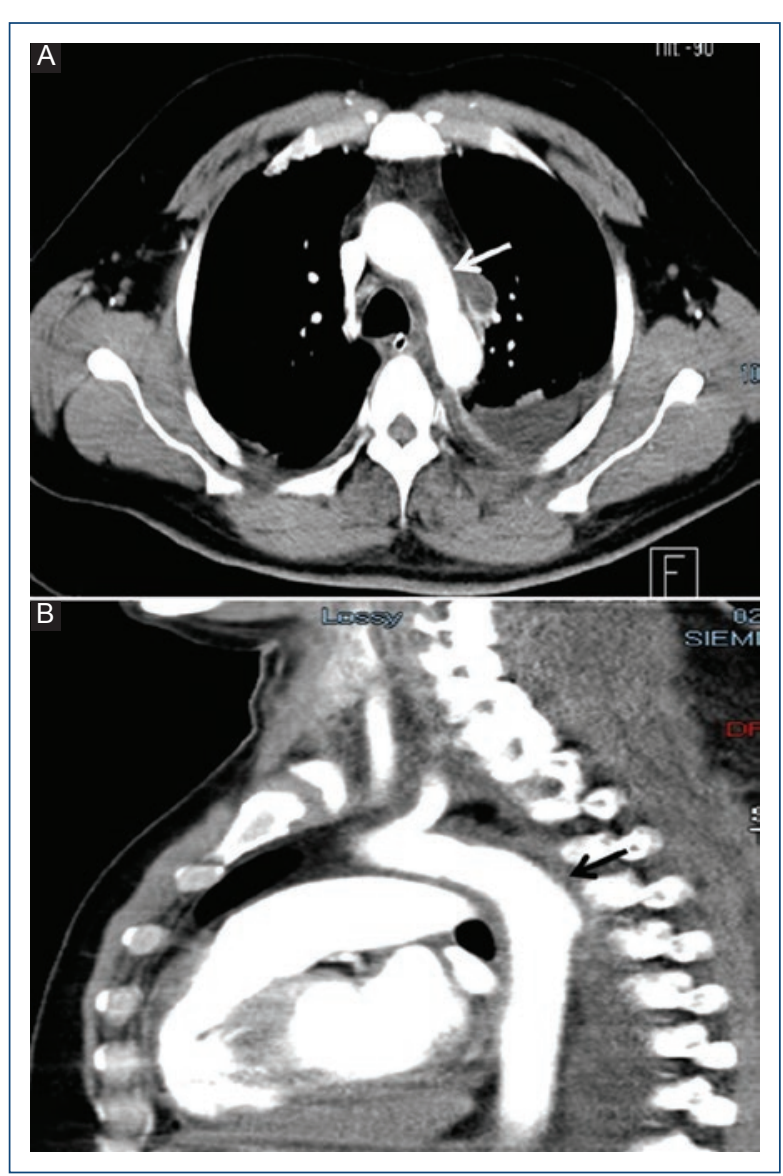

Figura 2. Angiotomografía helicoidal con contraste en fase arterial, cortes axial (A) y sagital (B): en ambos se observa injerto interpuesto adecuadamente (flechas blanca y negra), con buena restauración de la continuidad aórtica y sin dilatación aórtica proximal o distal, así como reabsorción del hematoma periaórtico.

encontró que la reparación diferida fue asociada con menor mortalidad (RR: 2.07; IC 95\%: 1.03-4.15) y tasas de paraplejia significativamente reducidas (RR: 5.90; IC 95\%: 1.51-22.97). Aunque no hay punto de corte con respecto al tiempo que pasa entre la recepción hospitalaria del paciente y la cirugía de reparación que delimite los conceptos de «inmediato» $y$ «tardío» (Whal, et al. ${ }^{8}$ consideran reparación tardía cuando han pasado más de 12 horas, Hemmila, et al. ${ }^{9}$ hablan de reparación tardía cuando han pasado más de 16 horas), la tendencia actual es utilizar la terapia hipotensora controlada para planear la cirugía de reparación, y evitar un procedimiento inmediato, incluso en pacientes donde no hay lesiones asociadas que comprometan la vida. En nuestro centro de atención se ha optado por el manejo tardío siempre que sea posible (nunca dentro de las primeras 6 horas de ingreso del paciente al hospital).
En el 2008, se publica el reporte que compara la práctica clínica y resultados entre un estudio multicéntrico prospectivo, completado en 1997 (AAST1) y otro similar completado en 2007 (AAST2). Entre los dos estudios se observaron tres cambios principales: primero, que el tiempo transcurrido desde la admisión del paciente a la reparación definitiva incrementó significativamente desde el AAST1 al AAST2 (16.5 \pm 70.8 h vs. $54.6 \pm 106.6$ h; $p<0.001)$; segundo, la tasa de paraplejia como complicación relacionada con el procedimiento se redujo, y tercero, el método de reparación definitiva pasó de ser con técnicas exclusivamente abiertas en 1997 a predominantemente endovasculares en 2007, ya que casi el $65 \%$ de los pacientes fueron manejados con esta técnica ${ }^{10}$. Este último estudio mostró una disminución significativa de la mortalidad temprana con la técnica endovascular en comparación con la reparación abierta en pacientes con o sin lesiones mayores asociadas. Por otro lado, la incidencia de complicaciones serias relacionadas con el procedimiento endovascular fue del $20 \%$. El seguimiento a largo plazo todavía no es suficiente para describir las fallas o complicaciones que pudieran desarrollarse con este abordaje. Demetriades, et al. ${ }^{11}$ mencionan la fuga del stent como principal complicación, sobre todo en pacientes jóvenes, sin embargo, las innovaciones a los dispositivos están en desarrollo y en un hospital de bajo volumen como el nuestro quizá sea más difícil el desarrollo de dicha habilidad.

\section{Conclusión}

El uso de antihipertensivos es mandatorio en todos los pacientes con sospecha de lesión aórtica por trauma, ya que reducen el riesgo de ruptura y permiten la estabilización y evaluación de otras lesiones. El uso de perfusión aórtica distal por medio de bypass o derivación temporal aurícula-arteria femoral izquierda disminuye el riesgo de paraplejia en aquellos pacientes en que se opte por una reparación abierta.

\section{Conflicto de intereses}

Ninguno.

\section{Financiamiento}

Ninguno. 


\section{Responsabilidades éticas}

Protección de personas y animales. Los autores declaran que para esta investigación no se han realizado experimentos en seres humanos ni en animales.

Confidencialidad de los datos. Los autores declaran que han seguido los protocolos de su centro de trabajo sobre la publicación de datos de pacientes.

Derecho a la privacidad y consentimiento informado. Los autores han obtenido el consentimiento informado de los pacientes y/o sujetos referidos en el artículo. Este documento obra en poder del autor de correspondencia.

\section{Bibliografía}

1. Gombert A, Barbati ME, Storck M, Kotelis D, Keschenau P, Pape HC. Treatment of blunt thoracic aortic injury in Germany-Assesment of the TraumaRegister DGU. PLoS One. 2017;12:e0171837.

2. Lee WA, Matsumura JS, Mitchell RS, Farber MA, Greenberg RK, Azizzadeh $A$, et al. Endovascular repair of traumatic thoracic aortic injury: clinical practice guidelines of the Society for Vascular Surgery. J Vasc Surg. 2011;53:187-92.

3. De Mestral C, Dueck A, Sharma SS, Haas B, Gomez D, Hsiao M. Evolution of the incidence, management, and mortality of blunt thoracic aortic injury: a population-based analysis. J Am Coll Surg. 2013;216: 1110-5.

4. Antonopoulos CN, Sfyroeras GS, Kallinis A, Kakisis JD, Liapis CD, Petridou ET. Epidemiology of concomitant injuries in traumatic thoracic aortic rupture: a meta-analysis. Vascular. 2014; 22:395-405.

5. Cullen E, Lantz E, Johnson C, Young P. Traumatic aortic injury: CT findings, mimics, and therapeutic options. Cardiovasc Diagn Ther. 2014; 4:238- 44

6. Fabian TC, Davis KA, Gavant ML, Croce MA, Melton SM, Patton JH Jr, et al. Prospective study of blunt aortic injury: helical CT is diagnostic and hypertensive therapy reduces rupture. Ann Surg. 1991;227:666-76.

7. Fox N, Schwartz D, Salazar JH, Haut ER, Dahm P, Black JH, et al. Evaluation and management of blunt traumatic aortic injury: a practice management guideline from the Eastern Association for the Surgery of Trauma. J Trauma Nurs. 2015;22:99-110.

8. Wahl WL, Michaels AJ, Wang SC, Dries DJ, Taheri PA. Blunt thoracic aortic injury: delayed or early repair? J Trauma. 1999;47:254-9.

9. Hemmila MR, Arbabi S, Rowe SA, Brandt MM, Wang SC, Taheri PA, et al. Delayed repair for blunt thoracic aortic injury: is it really equivalent to early repair? J Trauma. 2004;56:13-23.

10. Demetriades D, Velmahos GC, Scalea TM, Jurkovich GJ, Karmy-Jones R, Teiveira PG, et al. Diagnosis and treatment of blunt thoracic aortic injuries: changing perspectives. J Trauma. 2008;64:1415-8.

11. Demetriades D, Velmahos GC, Scalea TM, Jurkovich GJ, Karmy-Jones R, Teixeira PG, et al. Operative repair or endovascular stent graft in blunt traumatic thoracic aortic injuries: results of an American Association for the Surgery of Trauma Multicenter Study. J Trauma. 2008;64:561-70. 